\title{
THE EFFECT OF CONDUCTIVITY ON HOTSPOTS
}

\author{
N. F. SMYTH ${ }^{1}$
}

(Received 28 March 1991; revised 10 May 1991)

\begin{abstract}
In industrial applications of microwave heating, it has been observed that rather than the heating taking place uniformly, regions of high temperature, called hotspots, tend to form. Depending on the industrial application, these can be either desirable or undesirable, and hence a theoretical understanding of the properties of the material that lead to hotspot formation is necessary. It has been shown in previous studies that hotspot formation is a product of the nonlinear dependence of microwave energy absorption by the material on temperature. It is shown in the present work that the conductivity of the material can have a significant effect on hotspot formation and can, if large enough, stop a hotspot from forming.
\end{abstract}

\section{Introduction}

The use of microwave radiation for heating in industrial settings is finding more widespread application. With this increased use, a number of unexpected problems have been found to occur. These problems are due to the large temperature increases involved in some industrial applications of $\mathrm{mi}$ crowave heating, so that the temperature dependence of the properties of the material being heated, such as conductivity and microwave energy absorption, become important. Foremost amongst these problems is the phenomenon of a hotspot, which is a small region of very high temperature relative to its surroundings. Hotspots can reach such large temperatures that the material melts ([2]), which can be either desirable (metal smelting) or undesirable (drying, sintering), depending on the application.

Due to its industrial importance, the hotspot phenomenon has recently been subject to much theoretical analysis. Since the microwave heating of

\footnotetext{
${ }^{1}$ Department of Mathematics, The King's Buildings, University of Edinburgh, Mayfield Road, Edinburgh, Scotland, EH9 3JZ .

(C) Copyright Australian Mathematical Society 1992, Serial-fee code 0334-2700/92
} 
a material is governed by a coupling of electromagnetic and thermal phenomena, and furthermore, since the high temperatures involved in a hotspot mean that the temperature dependence of the properties of the material need to be included, the full analysis of microwave heating is quite difficult. However, as a hotspot occupies a small region, the simplifying assumption that the microwave radiation has constant amplitude in the region of the hotspot is made ([5], [6], [8], [12]). When this assumption is made, the temperature of the material is governed by the forced heat equation

$$
\frac{\partial T}{\partial t}=\nu \nabla^{2} T+\gamma(T),
$$

where $\gamma(T)$ is the (temperature dependent) rate of microwave energy absorption by the material (see [10], [11]). Equation (1.1) is also the reactiondiffusion equation which occurs in the theory of chemical reactions, with $\gamma$ being the temperature-dependent reaction rate (see, for example, [1], [3], [7]). Coleman [6] assumed that

$$
\gamma(T)=\gamma_{0} T^{\gamma_{1}}
$$

and with $\nu=0$, showed that hotspots will form if $\gamma_{1}>1$, hotspots manifesting themselves by the temperature becoming infinite in finite time. Furthermore, solutions $T(t)$ of $(1.1)$ are unstable to small perturbations if $\gamma_{1}>1$, the growth of small perturbations being associated with hotspots. However, temperature dependencies of the form (1.2) are unrealistic since $\gamma \rightarrow \infty$ as $T \rightarrow \infty$. Coleman [6] found numerically that for Arrhenius dependencies of the form

$$
\gamma=\gamma_{0} e^{-\gamma_{1} / T}
$$

and for $\nu$ sufficiently small, $T$ became large in finite time, corresponding to hotspot formation. It was further found that large values of $\nu$ were required to stop the formation of a hotspot.

Hill and Smyth [8] found steady-state solutions of (1.1) in planar and cylindrical geometries for exponential dependencies

$$
\gamma=\gamma_{0} e^{\gamma_{1} T}
$$

with $\nu$ constant and $T=T_{0}$ on the boundary of the body. Such solutions were found to be multi-valued, with two possible steady states. The steady state of lower temperature was found to be stable and the other steady state unstable to small perturbations. For $T_{0}>f\left(\nu /\left(\gamma_{0} \gamma_{1} a^{2}\right)\right)$, where $f$ is a function which was determined, no steady-state solutions existed and it was found numerically that a hotspot formed in these cases.

Roussy et al [12] solved (1.1) numerically for a cylindrical body with $\gamma$ quadratic in temperature and a convective heat-loss boundary condition. They then found an approximate criterion for a hotspot to form. Brodwin 
et al [5] found steady-state solutions of (1.1) for a thin slab with a convective and radiative heat-loss boundary condition. They found that the steady state was a multivalued function of the incident amplitude of the microwave field. The branch of lower temperature is stable until a critical temperature is reached, whereupon the second branch, corresponding to unstable solutions, folds back. For certain types of dependencies of $\gamma$ on $T$, a point is reached where this second branch again increases as the temperature is increased. The corresponding steady state is stable, is generally of high temperature, and so corresponds to a hotspot.

The present work is concerned with extending the results of [8] to include the effects of the conductivity of the material and temperature-dependent thermal diffusivity $\nu$. The conductivity of the material causes the microwave radiation to decay as it propagates through the material. It is found that for large enough values of the conductivity, a hotspot will not form, as expected on physical grounds. The dependence of this critical value of the conductivity on the other parameters in the case of (1.4) is determined.

\section{Governing equation}

The equations governing the microwave heating of a material are the damped wave equation for the electric field associated with the microwave radiation

$$
E_{t t}+\sigma(T) E_{t}=c_{1}^{2}(T) \nabla^{2} E
$$

and the forced heat equation

$$
T_{t}=\nu(T) \nabla^{2} T+\gamma(T)|E|^{2}
$$

governing the resultant heat flow, where $|E|$ denotes the amplitude of the electric field ([10], [11]). The parameter $\sigma$ is proportional to the conductivity of the material, $\gamma$ is the rate at which microwave energy is taken up by the material and $c_{1}$ is the microwave speed. The damped wave equation (2.1) can be derived from Maxwell's equations under the assumption that $\sigma$ is small or that $c_{1}$ is temperature independent and $\sigma$ is slowly-varying ([10], [11]). If $\sigma$ is $0(1)$ and $c_{1}$ is temperature dependent, then the equations for the electric and magnetic fields are coupled and of a more involved form than (2.1). In physical situations, $\gamma \propto \sigma$ for constant $c_{1}$ ([11]). In the present work, we shall take $\sigma$ constant and $\gamma$ temperature dependent. While this gives an unphysical temperature variation for $\sigma$, solving (2.1) for temperature dependent $\sigma$ is difficult. Furthermore, taking $\sigma$ constant should give an indication of the effect of a physical $\sigma$ on the formation of hotspots, which is the central concern of the present work. For temperature 
dependent thermal diffusivity, it is usual to take (2.2) in the form

$$
T_{t}=\nabla \cdot(\nu(T) \nabla T)+\gamma(T)|E|^{2} .
$$

If we assume the power-law dependencies

$$
\begin{aligned}
& \nu=\nu_{0} T^{\nu_{1}}, \\
& \gamma=\gamma_{0} T^{\gamma_{1}}
\end{aligned}
$$

then setting

$$
\bar{T}=T^{1+\nu_{1}}
$$

results in (2.3) becoming

$$
\bar{T}_{t}=\bar{\nu}(\bar{T}) \nabla^{2} \bar{T}+\bar{\gamma}(\bar{T})|E|^{2},
$$

where

$$
\begin{gathered}
\bar{\nu}=\nu_{0} \bar{T}^{\nu_{1} /\left(1+\nu_{1}\right)}, \\
\bar{\gamma}=\gamma_{0}\left(1+\nu_{1}\right) \bar{T}^{\left(\nu_{1}+\gamma_{1}\right) /\left(1+\nu_{1}\right)} .
\end{gathered}
$$

The transformed equation (2.6) is then of the form (2.2) with power-law dependencies for $\gamma$ and $\nu$. However, if the exponential dependencies

$$
\begin{aligned}
& \nu=\nu_{0} e^{\nu_{1} T}, \\
& \gamma=\gamma_{0} e^{\gamma_{1} T}
\end{aligned}
$$

are assumed, then setting

$$
\bar{T}=e^{\nu_{1} T}
$$

results in (2.3) becoming

$$
\bar{T}_{t}=\bar{\nu}(\bar{T}) \nabla^{2} \bar{T}+\bar{\gamma}(\bar{T})|E|^{2},
$$

where

$$
\begin{gathered}
\bar{\nu}=\nu_{0} \bar{T}, \\
\bar{\gamma}=\gamma_{o} \nu_{1} \bar{T}^{1+\gamma_{1} / \nu_{1}} .
\end{gathered}
$$

The transformed equation (2.10) is not of the form (2.2) with exponential dependencies for $\gamma$ and $\nu$. The forced heat equations (2.2) and (2.3) can then be transformed into each other with similar dependencies of $\gamma$ and $\nu$ on $T$ if this dependence is of a power-law form, but not if it is of an exponential form. The form (2.2) is chosen in the present work as this form leads to equations which can be solved exactly.

In the present work, we shall assume that $\sigma$ and $c_{1}$ are constant and that $\gamma$ and $\nu$ have the exponential dependencies (2.8). The exponential dependence of $\gamma$ on $T$ was also assumed by [8]. Von Hippel [13] gives extensive tables of experimental data for the dependence of the dielectric properties of various materials on temperature. While power-law dependencies of the form (2.4) 
fit a wider range of materials, the exponential dependencies (2.8) can be used for many materials. In particular, exponential dependencies have been used to model the behaviour of various ceramic materials ([4], [9], [14]). Let us assume that the microwave radiation incident on the material has an electric field of constant amplitude $E_{0}$ and that $a$ is a characteristic dimension of the body being heated. We then non-dimensionalise (2.1) and (2.2) by

$$
\begin{aligned}
E & =E_{0} u, \\
T & =\gamma_{1}^{-1} \theta, \\
x & =a \xi, \\
t & =\frac{a^{2}}{\nu_{0}} \tau .
\end{aligned}
$$

The non-dimensional governing equations are then

$$
\begin{gathered}
u_{\tau \tau}+s u_{\tau}=c^{2} \nabla^{2} u, \\
\theta_{\tau}=e^{\mu_{1} \theta} \nabla^{2} \theta+\Gamma e^{\theta}|u|^{2},
\end{gathered}
$$

where

$$
\begin{aligned}
s & =\frac{a^{2} \sigma}{\nu_{0}}, \\
c & =\frac{a c_{1}}{\nu_{0}}, \\
\mu_{1} & =\frac{\nu_{1}}{\gamma_{1}}, \\
\Gamma & =\gamma_{0} \gamma_{1} a^{2} E_{0}^{2} / \nu_{0} .
\end{aligned}
$$

The damped wave equation (2.13) has the damped travelling-wave solution

$$
u=e^{-k_{i} \xi} e^{i\left(k_{r} \xi-\omega \tau\right)},
$$

where

$$
k_{r}^{2}=\frac{\omega^{2}}{2 c^{2}}\left[1+\left(1+\frac{s^{2}}{\omega^{2}}\right)^{1 / 2}\right], k_{i}^{2}=\frac{\omega^{2}}{2 c^{2}}\left[-1+\left(1+\frac{s^{2}}{\omega^{2}}\right)^{1 / 2}\right]
$$

It can be seen from (2.17) that large values of the conductivity $s$ result in the rapid decay of the electric field away from $\xi=0$, so that the electric field is non-zero only in a boundary layer at $\xi=0$. To be specific, let us assume that we have a one-dimensional planar body in the region $0 \leq \xi \leq 1$ and that microwave radiation of amplitude $|u|=1$ is incident on the slab at $\xi=0$. Then the microwave field in the body is given by (2.16) if any 
reflections at the surface $\xi=1$ are ignored. With the electric field given by (2.16), the forced heat equation (2.14) becomes

$$
\frac{\partial \theta}{\partial \tau}=e^{\mu_{1} \theta} \frac{\partial^{2} \theta}{\partial \xi^{2}}+\Gamma e^{\theta-2 k_{i} \xi}
$$

\section{Steady-state solutions}

In this section, we shall seek steady-state solutions of the forced heat equation (2.18), as was done by [8]. The absence of such solutions indicates the conditions under which a hotspot will form. Such steady-state solutions are possible for boundary conditions which involve heat loss to the surroundings, such as the fixed temperature boundary conditions to be used here. Insulated boundaries will not yield a steady state. If we define

$$
\boldsymbol{\Theta}=\left(1-\mu_{1}\right) \theta-2 k_{i} \xi,
$$

then the steady-state solutions of (2.18) satisfy

$$
\frac{\partial^{2} \Theta}{\partial \xi^{2}}+\left(1-\mu_{1}\right) \Gamma e^{\Theta}=0 \text {. }
$$

Hill and Smyth [8] considered this equation for $\mu_{1}=0$ (and $k_{i}=0$ ). Equation (3.2) has distinct solutions for $\mu_{1}>1$ and $\mu_{1}<1$. The solution for $\mu_{1}<1$ is similar to that of [8] and it may be found that

$$
\left(1-\mu_{1}\right) \theta=2 k_{i} \xi+\log \left[\frac{A}{2 \Gamma\left(1-\mu_{1}\right)} \operatorname{sech}^{2} \frac{\sqrt{A}}{2}(\beta+\xi)\right] \text {. }
$$

For $\mu_{1}>1$, it may be shown that there are two possible solutions

$$
\left(1-\mu_{1}\right) \theta=2 k_{i} \xi+\log \left[\frac{A}{2 \Gamma\left(\mu_{1}-1\right)} \sec ^{2} \frac{\sqrt{A}}{2}(\beta+\xi)\right]
$$

and

$$
\left(1-\mu_{1}\right) \theta=2 k_{i} \xi+\log \left[\frac{A}{2 \Gamma\left(\mu_{1}-1\right)} \operatorname{cosech}^{2} \frac{\sqrt{A}}{2}(\beta+\xi)\right] .
$$

The solution (3.4b) is possible if $\beta$ and $\beta+1$ have the same sign.

Let us first consider the solution for $\mu_{1}<1,(3.3)$. The fixed-temperature boundary condition $\theta=\theta_{0}$ at $\xi=0,1$ gives

$$
\frac{A}{2 \Gamma\left(1-\mu_{1}\right)} \operatorname{sech}^{2} \frac{\sqrt{A}}{2} \beta=e^{\left(1-\mu_{1}\right) \theta_{0}}
$$


and

$$
\frac{A}{2 \Gamma\left(1-\mu_{1}\right)} \operatorname{sech}^{2} \frac{\sqrt{A}}{2}(\beta+1)=e^{\left(1-\mu_{1}\right) \theta_{0}-2 k_{i}} .
$$

The relations (3.5) can be shown to yield

$$
\frac{2 \Gamma\left(1-\mu_{1}\right)}{A} e^{\left(1-\mu_{1}\right) \theta_{0}} \sinh ^{2} \frac{\sqrt{A}}{2}=2 e^{k_{i}} \cosh \frac{\sqrt{A}}{2}-1-e^{2 k_{i}},
$$

which determines $A$, so that $\beta$ follows from (3.5a). For $\mu_{1}=0$ and $k_{i}=0$, it may be shown that (3.6) is the same as the equivalent expression of [8]. For $A=0$, the left-hand side of (3.6) is positive (and finite) and the righthand side of (3.6) is negative. Also as $A \rightarrow \infty$, the left-hand side of (3.6) dominates over the right-hand side. Hence if (3.6) has one solution, then it must have two solutions.

It was shown by [8] that for $\mu_{1}=0$ and $k_{i}=0,(3.6)$ has two solutions if

$$
\theta_{0} \leq \log \left[3.5138 \Gamma^{-1}\right]
$$

in the present non-dimensionalisation, and no solutions otherwise. In the limit $k_{i} \gg 1$, the dominant terms of (3.6) are

$$
2 e^{k_{i}} \cosh \frac{\sqrt{A}}{2}-e^{2 k_{i}}=0,
$$

so that one solution is, to first order,

$$
A=4 k_{i}^{2} \text {. }
$$

From (3.5a), we then have for $k_{i} \gg 1$,

$$
\beta= \pm k_{i}^{-1} \operatorname{sech}^{-1}\left[\frac{\Gamma\left(1-\mu_{1}\right)}{2 k_{i}^{2}} e^{\left(1-\mu_{1}\right) \theta_{0}}\right]^{1 / 2} .
$$

The solution (3.9) and (3.10) exists for any value of $\theta_{0}$ (since $k_{i} \gg 1$ ), so that a steady state exists for all $\theta_{0}$ for $k_{i} \gg 1$. Hence a hotspot will not form for $k_{i} \gg 1$ i.e. for large conductivity $s$ (see 2.17). This is to be' expected as large values of $k_{i}$ (i.e. conductivity) cause the electric field to decay rapidly away from $\xi=0$. As the electric field is then weak in the body of the material, little heating can occur there and so a hotspot cannot form.

There is thus a value of $k_{i}, k_{i c}$ say, for which (3.6) always has a solution for $k_{i}>k_{i c}$, so that no hotspot will form for $k_{i}>k_{i c}$. The marginal case for (3.6) occurs when the two roots for $A$ coincide. In this case, we have from (3.6) that

$e^{k_{i}}=-4 \Gamma\left(1-\mu_{1}\right) A^{-3 / 2} e^{\left(1-\mu_{1}\right) \theta_{0}} \sinh \frac{\sqrt{A}}{2}+2 \Gamma\left(1-\mu_{1}\right) A^{-1} e^{\left(1-\mu_{1}\right) \theta_{0}} \cosh \frac{\sqrt{A}}{2}$ 
is also satisfied. It is found numerically that (3.6) and (3.11) have a solution if $k_{i}<17.65$. Hence $k_{i c}=17.65$ and no hotspot forms for $k_{i}>k_{i c}$.

The solution (3.3) is shown in Figure 1 for $k_{i}=0,1,3$ and $\mu_{1}=0, \Gamma=$ $1, \theta_{0}=1$. It can be seen that as $k_{i}$ increases, the temperature decreases and the temperature maximum becomes more skewed towards $\xi=0$. The overall temperature decreases as $k_{i}$ (i.e. conductivity $s$ ) increases since increasing values of $k_{i}$ result in more rapid decay of the electric field away from $\xi=0$, resulting in decreasing total heat input. Also the more rapid decay of the electric field away from $\xi=0$ causes more heating near $\xi=0$ and hence the skewness of the resulting temperature profile. As $\mu_{1}$ is decreased from zero, the temperature increases uniformly due to the diffusivity in (2.14) decreasing with increasing temperature for $\mu_{1}<0$.

Let us now consider the solution (3.4) for $\mu_{1}>1$. The fixed temperature boundary condition $\theta=\theta_{0}$ at $\xi=0,1$ gives

$$
\frac{A}{2 \Gamma\left(\mu_{1}-1\right)} \sec ^{2} \frac{\sqrt{A}}{2} \beta=e^{\left(1-\mu_{1}\right) \theta_{0}}
$$

and

$$
\frac{A}{2 \Gamma\left(\mu_{1}-1\right)} \sec ^{2} \frac{\sqrt{A}}{2}(\beta+1)=e^{\left(1-\mu_{1}\right) \theta_{0}-2 k_{i}}
$$

$\boldsymbol{\theta}$

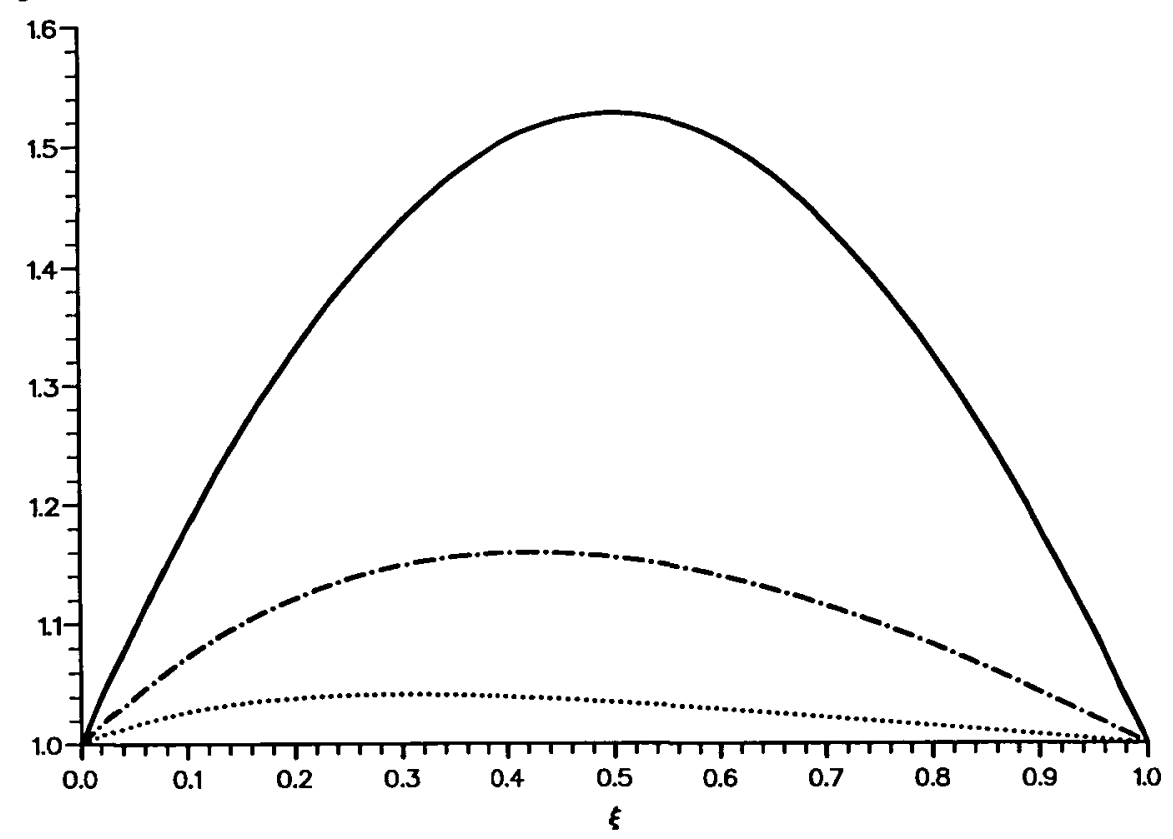

Figure 1. The solution (3.3) for $\mu_{1}=0, \Gamma=1, \theta_{0}=1$ and $k_{i}=0(-), 1(-\cdot-\cdot-)$, $3(\cdots \cdots)$. 
for (3.4a) and

$$
\frac{A}{2 \Gamma\left(\mu_{1}-1\right)} \operatorname{cosech}^{2} \frac{\sqrt{A}}{2} \beta=e^{\left(1-\mu_{1}\right) \theta_{0}}
$$

and

$$
\frac{A}{2 \Gamma\left(\mu_{1}-1\right)} \operatorname{cosech}^{2} \frac{\sqrt{A}}{2}(\beta+1)=e^{\left(1-\mu_{1}\right) \theta_{0}-2 k_{i}}
$$

for (3.4b). Again these equations may be examined in the limits $k_{i}=0$ and $k_{i} \gg 1$.

For $k_{i}=0$, it may be found from (3.12) and (3.13) that

$$
\beta=-\frac{1}{2}
$$

and for (3.12) that

$$
A=2 \Gamma\left(\mu_{1}-1\right) e^{\left(1-\mu_{1}\right) \theta_{0}} \cos ^{2} \frac{\sqrt{A}}{4} .
$$

Since $\beta<0$ and $\beta+1>0$, the solution (3.4b) is not valid for $k_{i}=0$. It can easily be seen that (3.15) has at least one solution for $A$ for all values of $\Gamma, \mu_{1}>1$ and $\theta_{0}$. In fact, for

$$
16 n^{2} \pi^{2}<2 \Gamma\left(\mu_{1}-1\right) e^{\left(1-\mu_{1}\right) \theta_{0}}<16(n+1)^{2} \pi^{2},
$$

there are $2 n+1$ distinct solutions for $A$. Hence a hotspot will not form for $\mu_{1}>1$ and $k_{i}=0$. This is to be expected as $\mu_{1}>1$ means that heat diffusion dominates over heat generation as $\theta \rightarrow \infty$, so that a hotspot cannot form as heat is rapidly diffused for large $\theta$.

Let us now consider the limit $k_{i} \gg 1$. Eliminating $\beta$ between both (3.12) and (3.13), we find

$$
2 \Gamma\left(\mu_{1}-1\right) A^{-1} \sin ^{2}\left(\frac{\sqrt{A}}{2}\right) e^{\left(1-\mu_{1}\right) \theta_{0}}=1+e^{2 k_{i}}-2 e^{k_{i}} \cos \frac{\sqrt{A}}{2}
$$

and

$$
2 \Gamma\left(\mu_{1}-1\right) A^{-1} \sinh ^{2}\left(\frac{\sqrt{A}}{2}\right) e^{\left(1-\mu_{1}\right) \theta_{0}}=1+e^{2 k_{i}}-2 e^{k_{i}} \cosh \frac{\sqrt{A}}{2}
$$

respectively. For $k_{i} \gg 1$, it can be found that (3.17) does not have a solution for $A$. However, for $k_{i} \gg 1$, it may be found in a similar manner to (3.9) that

$$
A=4 k_{i}^{2} \text {. }
$$

It may then be found from (3.13) that for $k_{i} \gg 1$,

$$
\beta=\left(2 k_{i}\right)^{-1} \log \left[\frac{8 k_{i}^{2}}{\Gamma\left(\mu_{1}-1\right)}\right]-\frac{\left(1-\mu_{1}\right) \theta_{0}}{2 k_{i}} .
$$


We thus see that there is one steady state for $\theta$ for $\mu_{1}>1$ and $k_{i} \gg 1$, so that no hotspot will form for $\mu_{1}>1$ and $k_{i} \gg 1$. For $\mu_{1}>1$, it then appears that no hotspot will form, which is expected as in this case thermal diffusion dominates over heat production.

The graphs of the solutions (3.4) are similar to those of the solution (3.3), examples of which are shown in Figure 1, with the temperature decreasing uniformly as $\mu_{1}$ is increased from 1 , this being due to the diffusivity in (2.14) increasing with increasing temperature for $\mu_{1}>1$. This can be seen in Figure 2, where the solution (3.4a) is shown fro $\mu_{1}=2,2.5,3$ and $\Gamma=1$, $\theta_{0}=1, k_{i}=0$.

In [8], a steady-state solution for $\theta$ was found in the case of a cylindrically symmetric body for $\mu_{1}=0$ and $k_{i}=0$. The cylindrically symmetric solution of the damped wave equation (2.13) corresponding to (2.16) is

$$
u=H_{0}^{(2)}(\lambda r) e^{-i \omega \tau} \text {, }
$$

where

$$
\lambda=\frac{\omega}{c}\left[1+\frac{i s}{\omega}\right]^{1 / 2}
$$

and $H_{0}^{(2)}$ is a Hankel function. Unfortunately, when (3.21) is substituted

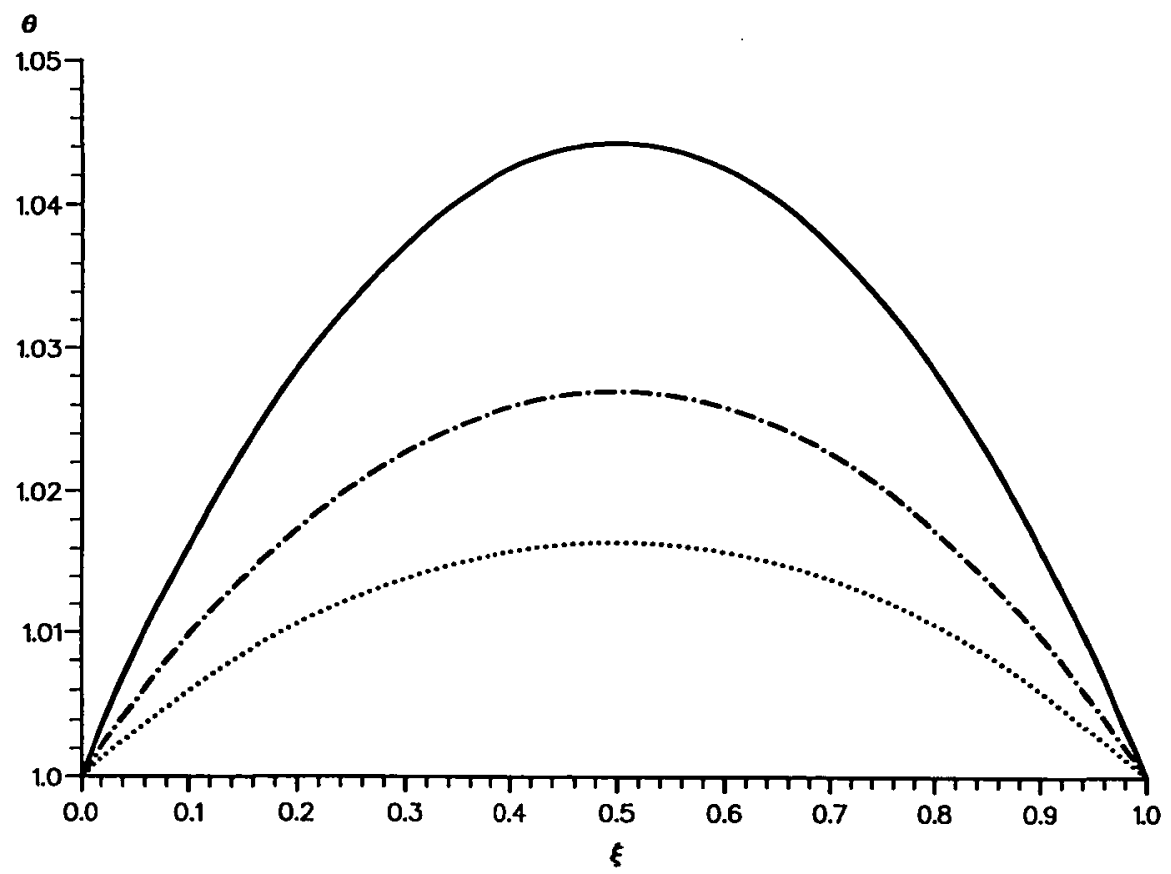

Figure 2. The solution (3.4a) for $k_{i}=0, \Gamma=1, \theta_{0}=1$ and $\mu_{1}=2(-), 2.5(-\cdot-\cdot-)$, $3(\cdots \cdots)$. 
into the forced heat equation (2.14), steady-state solutions for $\theta$ cannot be found. Presumably however, similar behaviour to the planar case would be found if such steady-state solutions could be determined.

\section{References}

[1] W. F. Ames, Nonlinear partial differential equations in engineering, Vol.I, (Academic Press, New York, 1965).

[2] J. C. Araneta, M. E. Brodwin and G. A. Kriegsmann, "High temperature microwave characterisation of dielectric rods", IEEE MTT - 32 (1984) 1328-1336.

[3] $\mathrm{R}$. Aris, The mathematical theory of diffusion and reaction in permeable catalysts, Vol. 1, the theory of the steady state, (Oxford University Press, Oxford, 1975).

[4] A. S. Barker, J. A. Ditzenberg and J. P. Remeika, "Lattice vibrations and its transport spectra in $\beta$-alumina II: microwave spectra”, Phys. Rev. B. 14 (1976) 4254-4265.

[5] M. E. Brodwin, G. A. Kriegsmann and D. G. Watters, "Temperature instability in the microwave heating of a uniformly illuminated planar slab", IEEE to appear (1991).

[6] C. J. Coleman, "On the microwave hotspot problem", J. Austral. Math. Soc. Ser. B 33 (1991).

[7] D. A. Frank-Kamenetskii, Diffusion and heat exchange in chemical kinetics, (Princeton University Press, Princeton, New Jersey, 1955).

[8] J. M. Hill and N. F. Smyth, "On the mathematical analysis of hotspots arising from microwave heating", Math Engng. in Industry 2(4) (1990) 267-278.

[9] W. D. Kingery, H. K. Bowen and D. R. Uhlman, Introduction to ceramics (John Wiley, New York, 1976).

[10] A. S. Metaxas and R. J. Meredith, Industrial microwave heating (IEE Power Engineering Series, 4, P. Peregrinus on behalf of the Institution of Electrical Engineers, London, 1983).

[11] A. H. Pincombe and N. F. Smyth, "Microwave heating of materials with low conductivity", Proc. Roy. Soc. Lond. A, 433 (1991) 479-498.

[12] G. Roussy, A. Bennani and J. Thiebaut, "Temperature runaway of microwave irradiated materials", J. Appl. Phys. 62 (1987) 1167-1170.

[13] A. R. von Hippel, Dielectric materials and applications (MIT Press, Cambridge, MA, 1954).

[14] W. Wo, "Millimeter wave dielectric property measurements of gyration window materials", ORNL Sub-83-51926, Vols. 1-2, Final report 1985-86. 